\title{
Local treatment enhances the efficacy of ADT
}

While androgen deprivation therapy (ADT) remains a mainstay of the management of advanced and aggressive prostate cancer, the optimum timing and application of this treatment modality has yet to be determined. In response, a systematic review team from Belgium and The Netherlands has investigated whether local treatment affects the efficacy of ADT. "Several studies have shown that in certain circumstances the differences in clinical outcomes [for prolonged $\mathrm{ADT}$ ] are quite large," says Paul Verhagen, lead investigator on the study. Although a radiosensitizing effect of ADT is often mentioned, it is insufficient to explain all the observed differences in outcomes.

The investigators included published randomized trials of immediate versus deferred and adjuvant ADT for the treatment of various stages of prostate cancer-by orchiectomy or luteinizinghormone-releasing hormone analogs-in their analysis. All trials reported prostate cancer-specific and overall survival. Trials that used antiandrogens only, or estrogens, were excluded.

Overall, five studies compared immediate versus delayed ADT. On metaanalysis, the investigators found a modest benefit of immediate ADT in terms of both overall and prostate cancer-specific survival (hazard ratio [HR] $0.90,95 \% \mathrm{CI}$ 0.83-0.97; and HR 0.79, 95\% CI 0.710.89 ; respectively). Only one trial showed a significant improvement in survival when considered individually.

A further five studies investigated the effects of ADT adjuvant to primary local therapy, which comprised radiotherapy in four trials, and radical prostatectomy in the other. Adjuvant ADT was associated with improved overall and prostate cancer-specific survival compared with primary therapy alone (HR 0.69, 95\% CI 0.61-0.79; and HR 0.51, 95\% CI 0.41-0.63; respectively). However, the investigators noted substantial variation in the results of these trials, particularly in prostate cancer-specific mortality.

"Prolonged exposure to ADT improves survival in patients with aggressive disease," concludes Verhagen, " but only after radical prostatectomy or irradiation of the primary tumor." These results indicate that local therapy is an important component of treatment, even in patients with advanced incurable cancer. Both ADT and local therapy, however, are associated with adverse effects, and a challenge will be to identify the subset of patients who will benefit most from such an aggressive treatment approach.

\section{Nick Groves-Kirkby}

Original article Verhagen, P. C. M. S. et al. Does local treatment of the prostate in advanced and/or lymph node metastatic disease improve efficacy of androgendeprivation therapy? A systematic review. Eur. Urol. doi:10.1016/j.eururo.2010.05.027 